\title{
Alterstice
}

Revue internationale de la recherche interculturelle

International Journal of Intercultural Research

Revista International de la Investigacion Intercultural

\section{Introduction à la psychologie interculturelle, de Laurent Licata et Audrey Heine}

\section{Bertrand Troadec}

Volume 3, numéro 1, 2013

URI : https://id.erudit.org/iderudit/1077501ar

DOI : https://doi.org/10.7202/1077501ar

Aller au sommaire du numéro

Éditeur(s)

Alterstice

ISSN

1923-919X (numérique)

Découvrir la revue

Citer ce compte rendu

Troadec, B. (2013). Compte rendu de [Introduction à la psychologie

interculturelle, de Laurent Licata et Audrey Heine]. Alterstice, 3(1), 85-91.

https://doi.org/10.7202/1077501ar d'utilisation que vous pouvez consulter en ligne.

https://apropos.erudit.org/fr/usagers/politique-dutilisation/ 


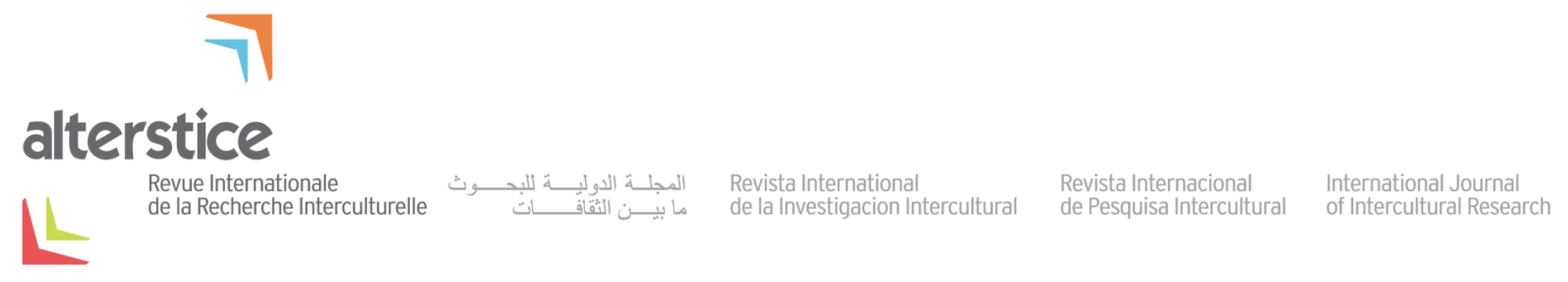

NOTE DE LECTURE

\title{
Introduction à la psychologie interculturelle, de Laurent Licata et Audrey Heine
}

\author{
Bertrand Troadec ${ }^{1}$
}

\author{
Rattachement de l'auteur \\ ${ }^{1}$ Université des Antilles et de la Guyane, pôle Martinique
}

\section{Correspondance}

bertrand.troadec@martinique.univ-ag.fr

\section{Références de l'ouvrage :}

Licata, L. et Heine, A. (2012). Introduction à la psychologie interculturelle. Bruxelles : De Boeck Université, 336 p.

\section{Pour citer cet article :}

Troadec, B. (2013). Introduction à la psychologie interculturelle [note de lecture]. Alterstice, 3(1), 85-91.

\section{Présentation de l'ouvrage}

S'il est une entrée en matière plutôt originale et inédite pour un ouvrage sur la psychologie interculturelle, rédigé en français, c'est bien le pluriel, utilisé par les auteurs, Laurent Licata et Audrey Heine (2012), pour caractériser leur objectif principal qui est de présenter «les psychologies interculturelles » (p. 7). De plus, cette Introduction à la psychologie interculturelle est " encadrée » par une préface rédigée par John Berry et une postface rédigée par Pierre Dasen. II s'agit là d'un parrainage intellectuel de " poids » quand on connaît l'importance de la contribution de chacun des deux auteurs au développement de la psychologie interculturelle dans le monde contemporain.

Cela dit, dans sa préface, Berry (re)définit la psychologie interculturelle comme étant l'étude de la relation entre le contexte culturel et le comportement humain. Cette relation apparaît implicitement causale dans le sens suivant (je le cite): "la culture influence le comportement» (p.5), car elle ressortit au paradigme explicatif qui domine actuellement la science occidentale et qui se mondialise rapidement. Ce paradigme est dit " expérimental », sinon "quasi expérimental». Il caractérise une partie de la psychologie, notamment interculturelle, comme de nombreuses autres sciences. Ce paradigme prend la forme de variables indépendantes [VI] ou " causes » (ici, la culture) et de variables dépendantes [VD] ou « conséquences » (ici, le comportement), fonction d'un schéma causal linéaire [VI $\Rightarrow$ VD].

II semble que Licata et Heine en fassent aussi leur propre définition (p. 18), bien que les auteurs ajoutent un troisième terme aux deux précédents, celui de « faits psychiques » (p. 30). En effet, si l'on veut éviter d'être critiqué de se prévaloir d'une sorte de "béhaviorisme méthodologique ", comme Michael Cole (1996) a pu le faire, il convient, à l'instar des cognitivismes, d'analyser aussi l'intérieur de la " boîte noire », c'est-à-dire le psychisme ou l'esprit (mind). Plus loin dans l'ouvrage, Licata et Heine rapportent par ailleurs que « Berry et al. (2011, p. 5) ont 
récemment défini la psychologie interculturelle, comparative cette fois, comme étant "l'étude : des similarités et des différences au niveau du fonctionnement psychologique individuel entre divers groupes culturels et ethnoculturels" » (p. 68).

Le fonctionnement psychologique ou les faits psychiques (facteur ou VI « interne »?) sont supposés jouer aussi un rôle important sur le comportement [VD] et non pas seulement la culture (facteur ou VI « externe »?). Mais, si la psychologie interculturelle modélise la culture certainement mieux que n'importe quelle "branche » de la psychologie, elle souffre parfois d'une faiblesse de clarté de la modélisation du psychisme ou de l'esprit (mind). De nos jours, ces modélisations varient considérablement en psychologie :

- Pour les plus classiques: la phénoménologie; le béhaviorisme; la théorie de la forme (gestalt); le structuralisme piagétien; le cognitivisme computationnel ou bien représentationnel ou fonctionnalisme; le connexionnisme;

- Pour les plus actuelles: le darwinisme neural-mental; l'évolutionnisme; la cognition située ou incarnée (situated cognition, embodiment); l'énaction; I'intentionnalité (intentionality); etc.

On peut considérer que le modèle écoculturel de Berry, dans le cadre plus général d'une cross-cultural psychology (ou psychologie interculturelle comparative) est à rattacher à une conception de l'esprit humain inspirée par le cognitivisme représentationnel (système de traitement d’information; compétences inférées; performances observées; etc.), alors que la conception "intentionnelle » de l'esprit humain à laquelle se réfère la cultural psychology (ou psychologie culturelle) de Richard Shweder (Stigler, Shweder et Herdt, 1990), par exemple, mais aussi celle de Jerome Bruner (1991), est d'inspiration phénoménologique. On ne peut donc pas les confondre et c'est probablement en ce sens qu'il y a bien des psychologies interculturelles, au pluriel. Les auteurs pouvaient alors oser un autre titre pour leur publication: Introduction aux psychologies interculturelles, mais le projet d'ouvrage n'aurait plus été le même.

\section{Partie 1 : Psychologie et culture}

Dans une première partie, Licata et Heine rappellent un postulat courant de la psychologie interculturelle, qui est que la culture " influence » la psychologie humaine, mais aussi que la psychologie humaine " façonne » la culture. Les deux entités sont définies comme étant ainsi en "interdépendance dynamique » (p. 32). Puis, les auteurs présentent en détail le modèle écoculturel de John Berry à l'appui de cette définition. Ce modèle correspond à la conception rappelée ci-dessus, selon laquelle la culture [VI] influence le comportement [VD]. Cette relation est ainsi représentée dans le modèle par des flèches essentiellement orientées de la gauche vers la droite [culture $\Rightarrow$ comportement]. Laurent Licata et Audrey Heine (2012) remarquent alors que "Berry et al. (2011) précisent récemment que, pour être complet, le modèle devrait comporter des flèches de droite à gauche »(p. 34), soit [culture $\diamond$ comportement] et donc, pour être tout à fait «complet», des flèches doubles: [culture $\Leftrightarrow$ comportement]. Pourquoi John Berry, Ype Poortinga, Seger Breugelmans, Athanasios Chasiotis et David Sam (2011) ne l'ont-ils donc pas fait?

De mon point de vue, cela entraînerait une modification radicale du paradigme scientifique utilisé qui ne pourrait plus reposer sur une conception linéaire de la causalité [VI $\Rightarrow$ VD], mais sur une conception systémique de celle-ci impliquant l'usage de concepts assez différents, tels que la survenance ou l'émergence. Certains défendent cet autre paradigme. Gustav Jahoda (2002) n'écrivait-il pas, en réaction au paradigme précédent, que la culture n'est pas la "cause » du comportement individuel? Elle " est " (dans un sens ontologique) le comportement qu'un individu "partage ", c'est-à-dire "négocie » et "adopte " (share en anglais), avec d'autres individus. Cette conception est revendiquée par la cultural psychology qui se démarque ainsi, de façon plus ou moins radicale, d'une cross-cultural psychology et de la méthode comparative, pour laquelle les analyses ne peuvent être faites que par une comparaison des effets moyens [VD] en fonction des différentes modalités de la VI-culture. À plusieurs reprises, j'ai tenté de trouver, au moins pour moi-même, une sortie de l'impasse qui obligerait à choisir l'une ou bien l'autre de ces deux attitudes, ontologique et épistémologique (Troadec, 2003, 2007 et 2011).

Alterstice - Revue Internationale de la Recherche Interculturelle, vol. 3, $n^{\circ} 1$ 
Il faut ajouter que d'autres auteurs modélisent aussi ce système à doubles flèches [culture $\Leftrightarrow$ comportement]. Par exemple, Pierre Dasen et Ramesh Mishra (2013) présentent un "modèle théorique intégré " (integrated theoretical framework) inspiré par plusieurs modèles antérieurs, tels que le "modèle systémique " (ecological systems theory) de Urie Bronfenbrenner, le modèle des "niches de développement " (developmental niches) de Charles Super et Sara Harkness et le « modèle écoculturel » (ecocultural model) de John Berry.

\section{Partie 2 : Identité et altérité}

La deuxième partie de l'ouvrage est une belle revue de la littérature à propos de la question de la conception de Soi, de la construction de l'identité ou de l'appartenance ethnique et culturelle, et du rapport à autrui. Peut-être aurait-on pu ajouter à cette revue des exemples de recherches empiriques détaillés, réalisées par la psychologie interculturelle sur cette problématique. Selon Licata et Heine, qui s'appuient sur les conceptions des spécialistes de la question, les différents aspects du Soi «forment souvent un tout relativement cohérent » (p. 79), ayant une continuité temporelle. Cela m'a fait immédiatement penser à l'argumentation de Francisco Varela, Evan Thompson et Eleanor Rosch (1993) à l'encontre de cette conception.

Dans le cadre d'une argumentation en faveur de la conception dite énactive de l'esprit (ou enaction), c'est-à-dire incarnée (embodied), Francisco Varela affirme que le Soi n'existe pas « en soi » (Varela, Thompson et Rosch, 1993). Rejoignant une autre conception, issue quant à elle d'une autre culture que la culture occidentale majoritaire, en l'occurrence le bouddhisme et sa méthodologie qu'est la méditation, il défend la thèse selon laquelle l'esprit (mind) est une entité non pas cohérente et stable dans le temps, mais au contraire instable, discontinue, temporaire, etc. Pour les trois auteurs, le Soi est donc un construit issu, entre autres, d'une tradition (cognitiviste) représentationaliste, produit par un observateur " extérieur » et qui l'infère en " troisième personne ". La réalité de l'expérience subjective propre à chacun, phénoménologique, vécue en " première personne ", si l'on peut dire de l'«intérieur», est que "l'esprit est dénué de soi » (p. 178). Cela ne signifie pas que l'individu n'ait pas de "structure cohérente" d'habitudes (p.179), mais cela signifie que la croyance en "une essence réelle, permanente, qui est à la source de notre identité » n'est pas fondée (ibidem).

L'hypothèse des auteurs est que la construction d'un Soi serait justement une "réponse émotionnelle à ce manque» (p. 180). Beaucoup de psychologues sociaux auraient sûrement du mal à accepter les assertions précédentes, étant persuadés de l'existence " en soi » du Soi qu'ils étudient. Pour Francisco Varela, Evan Thompson et Eleanor Rosch (1993), c'est parce que "notre culture [scientifique] est minée par le fait que nous sommes contraints, condamnés à croire en quelque chose [le Soi] dont nous savons que cela ne peut être vrai » (p. 185). (voir aussi l'ouvrage de Bault, Chambon, Maïonchi-Pino, Pénicaud, Putois et Roy, 2011)

Dans la suite de l'ouvrage, l'analyse du rapport à autrui est très intéressante, car elle permet une compréhension des fondements de l'ethnocentrisme occidental, sinon du racisme qu'il peut générer, et ses conséquences dans la vie de tous les jours, mais aussi dans la production de connaissance scientifique. Cela dit, qu'en est-il du point de vue des autres sur l'Occident? À propos de cette question, la situation évolue actuellement. Dans la préface de l'ouvrage Le renversement du ciel. Parcours d'anthropologie réciproque (Le Pichon et Sow, 2011), Umberto Eco définit ce qui a été dénommé, dans un premier temps, l'anthropologie alternative, et dans un second, l'anthropologie réciproque. Depuis de nombreux siècles, "les cultures se sont toujours observées mutuellement, mais en général nous, Occidentaux, n'avions connaissance que des observations que nous faisions sur les autres " (p. 9). Sans enlever à certains observateurs un "désir authentique " de comprendre les autres culturellement différents d'eux, Umberto Eco précise que l'anthropologie moderne a créé une "caste ", écrit-il, d'observateurs occidentaux qui s'estiment capables de comprendre les autres cultures (au sens "ethnique» du terme). En psychologie, la plupart des psychologues, de même que les psychologues dits culturels ou interculturels (dont je fais partie) s'estiment eux aussi capables de comprendre les autres, notamment leur psychisme. Mais il s'avère que les « autres » nous observent aussi !

Le projet Transcultura a consisté à demander à des hommes de culture (au sens de "cultivés"), sinon à des universitaires, d'Afrique ou de Chine ou d'ailleurs, de venir en Europe pour décrire, de leur point de vue, "nos » sociétés. De nos jours, du fait de la mondialisation de l'information via les journaux, la radio, la télévision, les sites 
internet, etc., tout le monde est au courant des réalités de tout le monde. II ne s'agit donc plus d'être confronté à l'étrangeté des autres, mais à leur différence ou diversité « au-delà des traits communs qui nous rattachent à l'espèce humaine » (Eco, dans Le Pichon et Sow, 2011, p. 10). La première phase d'anthropologie dite " alternative » a ainsi produit quelques résultats curieux, mais intéressants. Par exemple, "le conteur africain était frappé de stupéfaction de découvrir que les femmes françaises conduisaient à la laisse leurs chiens en promenade, ou stupéfait encore de voir comment les Européens, à la mer, se promenaient nus, chose qui pour un homme du continent noir révélait une absence totale de dignité » (p. 11).

On le comprend bien, il faut aller plus loin que ces observations des uns par les autres et vice-versa. L'anthropologie dite " réciproque » consiste alors à considérer « les uns et les autres comme des représentants de cultures diverses qui s'analysent face à face, ou montrent comment on peut réagir de façon différente devant les mêmes expériences " (Eco, dans Le Pichon et Sow, 2011, p. 12). II existe déjà quelques exemples remarquables de face à face culturel de ce genre, c'est-à-dire de réel dialogue interculturel, par exemple, relatifs à l'esprit et à ses propriétés, c'est-à-dire à l'objet de la psychologie. L'ouvrage, déjà " ancien », intitulé Passerelles, présente une série d'Entretiens avec le Dalaï-Lama sur les sciences de l'esprit (Hayward et Varela, 1995). Au cours de ces entretiens, les conceptions (telle celle du Soi) et les méthodes des sciences occidentales de l'esprit, dont la psychologie, et celles de la philosophie orientale bouddhiste sont mises face à face, permettant de discuter, voire de s'étonner, de ce qui leur est commun et différent. À l'instar d'une anthropologie réciproque, à quand une psychologie interculturelle « réciproque »?

\section{Partie 3 : La comparaison interculturelle}

Les deux auteurs de l'ouvrage portent un intérêt particulier à certains « objets » étudiés par la psychologie sociale, « objets » qui , certes, sont analysés ici selon une perspective interculturelle. Les domaines qui sont étudiés par la psychologie cognitive, par la psychologie clinique, par la psychologie du développement et autres sont donc peu présents. Mais cela est annoncé dès l'introduction.

Les travaux réalisés par Richard Nisbett et ses collaborateurs, qui, en comparant des groupes de sujets asiatiques et occidentaux, déterminent des "styles cognitifs " culturellement différents, en fonction des caractéristiques des environnements asiatiques et occidentaux, sont un bon exemple d'utilisation contemporaine de la méthode comparative (voir par exemple Nisbett, 2003). Ils méritent donc d'être présentés à un lectorat français. Sont aussi présentés les travaux réalisés par les développementalistes (Pierre Dasen, Patricia Greenfield, Heidi Keller, etc.), qui fournissent un autre exemple d'études interculturelles comparatives relatives à des « objets " tels que la genèse de la cognition, de la socialisation, des émotions, etc.

La discussion relative à l'emploi de la méthode comparative en psychologie interculturelle, qui est présentée à la fin de cette troisième partie de l'ouvrage, est digne d'intérêt. On a rappelé plus haut que cette méthode repose sur les exigences des analyses causales du paradigme quasi expérimental [VI $\Rightarrow$ VD] d'une science qui se veut explicative des phénomènes, et non pas simplement descriptive. Que ce paradigme soit " occidentalocentré " (Licata et Heine, 2012, p. 177), sinon même " étasuniocentré », quand on sait qu'il a été " fabriqué » par la science occidentale, est un truisme. Qu'il soit "imposé », parfois de façon sûrement "impérialiste ", à la science mondiale via l'usage généralisé et ségrégationniste de l'anglais (Hagège, 2012) est une réalité actuelle des formations universitaires, des publications scientifiques, des carrières des enseignants-chercheurs, etc., dans le monde entier. II est sûrement crucial de résister!

Cela dit, il faut bien disposer d'un moyen ou bien d'un autre pour étudier le réel, pour l'objectiver, et tout moyen est forcément centré "quelque part ». Le paradigme quasi expérimental est l'un de ces moyens. II est "préféré » par la science explicative. Comme l'a écrit Georges Devereux (1980), "les déformations ethnocentriques caractéristiques, dues à la culture à laquelle on appartient, sont inévitables. Plutôt que de les déplorer, nous devons en tenir compte, comme de sources d'erreur systématique » (p. 198). Pour y parvenir, il convient d'être le plus conscient possible du « moyen » d'investigation du réel dont on se sert et de ses limites. Comme je l'ai rappelé plus haut, c'est ce que j'ai tenté de faire et que je continue de faire, au moins pour moi (Troadec, 2003, 2007 et 2011). La réduction des « erreurs systématiques » dont parle Georges Devereux, ou plutôt leur prise de conscience,

Alterstice-Revue Internationale de la Recherche Interculturelle, vol. 3, $n^{\circ} 1$ 
car elles sont inévitables, est alors possible grâce au dialogue interculturel entre chercheurs, ou « discussion critique " (Popper, 1998). La collaboration de chercheurs de cultures différentes, dont les cultures qui font l'objet de la recherche, peut contribuer à cette prise de conscience, mais c'est prendre le risque d'en être transformé, c'est-à-dire métissé, et de devoir modifier en retour le "moyen » évoqué ci-dessus, c'est-à-dire le paradigme qui permet la production des connaissances scientifiques. Sans pour autant l'abandonner, à l'heure actuelle, je ne sais pas faire autrement.

Le dernier point de cette discussion relève de l'épistémologie des sciences humaines et sociales. Comme l'a écrit, par exemple, François Laplantine (1995) à propos de l'anthropologie, " nous ne sommes jamais des témoins objectifs observant des objets, mais des sujets observant d'autres sujets » (p. 169). Le sujet observateur, ethnocentré, utilisant un certain moyen d'analyser la réalité, lui aussi ethnocentré, produit - Jean Piaget disait « construit » - une connaissance relative aux sujets observés, c'est-à-dire l'objet de la psychologie interculturelle. Cette connaissance n'est jamais le réel " en soi » - Jean Piaget disait qu'il est une " limite jamais atteinte ", - elle est l'expression d'un réel construit "par soi ", sinon " par nous " les psychologues interculturels, et en est une approximation provisoire. Selon François Laplantine (1995), le travail de l'anthropologue, comme de tout autre observateur, consiste à " décider quels faits sont significatifs » (p. 193), c'est-à-dire à faire un tri parmi les données issues de l'observation, puis à avoir une " activité résolument théorique de construction [ou de modélisation] d'un objet qui n'existe pas dans la réalité » (idem).

L'exemple de la distinction quelque peu radicale, faite autrefois entre des sociétés individualistes (de l'indépendance des individus), d'une part, et des sociétés communautaristes (collectivist en anglais) (de l'interdépendance des individus), d'autre part, pris et discuté par les auteurs, est très éclairant. L'évolution de ce modèle initial, qui est rappelée dans le texte, exprimée d'abord en termes de deux pôles d'un même continuum, et non plus de deux entités distinctes, puis en termes de deux éléments d'une totalité plurielle, montre le caractère provisoire et évolutif de la connaissance scientifique.

Un autre problème fréquent est de finir « par tenir la construction de l'objet [le modèle] pour la réalité sociale ellemême. Or, la population que nous étudions ne nous a pas attendus pour attribuer des significations à ses pratiques » (Laplantine, 1995, p. 193). Il peut donc y avoir des divergences quant aux connaissances produites par l'un (le psychologue interculturel) et par les autres (ceux qu'il étudie) et même de l'étonnement. L'auteur estime que cette "inadéquation entre deux pensées" peut être "réduite en la traduisant dans un autre langage » (p. 194), en modifiant le modèle. Cela dit, c'est bien quelque chose de la culture scientifique occidentale, sinon mondiale, qui aura servi de moyen de le faire.

\section{Partie 4 : La psychologie des contacts interculturels}

Cette dernière partie de l'ouvrage est elle aussi fort intéressante. Elle met tout d'abord en évidence la complexité, peut-être faudrait-il parfois dire la complication, des concepts en jeu, sinon du lexique utilisé, lorsqu'il faut tenter d'objectiver comment un individu donné se fait une idée de lui-même (un Soi, une identité, une appartenance, etc.) fondée sur plusieurs «cultures. "Personnellement, je regrette que les assertions présentées par les deux auteurs, à partir de travaux publiés par des spécialistes du domaine, ne soient pas explicitement associées à un exposé de la méthodologie (entretiens, questionnaires, etc.) et des méthodes d'analyse des données utilisées par ces mêmes spécialistes (analyse de contenu, analyse en corrélations, analyse factorielle, etc.). II n'est pas impossible de penser que, pour une part, la diversité de ces assertions soit liée à la diversité des techniques de recueil des données et à celle des méthodes d'analyse, utilisés par les chercheurs, expliquant ainsi la complexité/complication de la situation conceptuelle et théorique.

Ensuite, le fait de présenter des travaux tout récents qui montrent que les discriminations qui sont issues du rapport de ce Soi aux autres (et pas seulement celles fondées sur l'appartenance pluriethnique ou multiculturelle) ont des effets négatifs sur la santé physique et psychique des personnes discriminées est à mettre au crédit des deux auteurs. Le sujet étant d'une importance capitale pour les sociétés actuelles, une clarification des concepts et des méthodes serait bienvenue. 
Au final, l'ouvrage rédigé par Laurent Licata et Audrey Heine (2012) est un ouvrage de qualité et important. L'objectif d'être une bonne Introduction à la psychologie interculturelle est atteint. D'un point de vue didactique, on apprécie les résumés en fin de chapitre et les quelques questions posées pour réfléchir le contenu du chapitre. On apprécie aussi les exemples de situations " concrètes ", originaux et attrayants. De plus, l'aspect historique de la psychologie interculturelle y est bien développé. L'organisation de l'ouvrage reste cependant assez classique. II est constitué d'une introduction et de quatre grandes parties (psychologie et culture; identité et altérité; comparaison interculturelle; psychologie des contacts interculturels), elles-mêmes constituées de plusieurs chapitres. Un autre aspect classique de l'ouvrage réside dans les "objets" qui sont majoritairement traités dans les recherches présentées et qui relèvent principalement de la psychologie sociale.

Il existe quand même, de nos jours, des perspectives qui apportent de la nouveauté en psychologie interculturelle. Les conceptions évolutionnistes de la culture, succinctement mentionnées dans l'ouvrage, en sont une (par exemple, Schaller, Norenzayan, Heine, Yamaqishi et Kameda, 2010). Mais, pour y adhérer, il faut se démarquer d'une vision caricaturale de l'évolutionnisme qui consiste à affirmer que cette perspective hiérarchise les cultures sur le continuum du progrès darwiniste, allant des moins évoluées, des moins développées, aux plus évoluées, aux plus développées. On n'en est plus là. Très récemment, il y a aussi une neuro-imagerie ou neuropsychologie interculturelle (transcultural neuroimagery) qui commence tout juste à montrer l'inscription de la diversité culturelle, mise en évidence par la psychologie interculturelle classique, «dans » l'organisation et le fonctionnement cérébral (par exemple, De Greck, Shi, Wang, Zuo, Yang, Wang, Northoff et Han, 2012).

Bref, parmi un ensemble d'universaux anthropologiques, dont la liste n'est pas «finie » et qui ne sont pas des réalités en soi, mais des potentialités, la culture (dont celle des enseignants-chercheurs en psychologie interculturelle) pourrait être la préférence pour certains d'entre eux (implicite la plupart du temps, car nous ne savons pas toujours ce que nous "préférons », mais qui peut devenir explicite). Cela n'exclut alors pas de modifier nos préférences, nos cultures, avec la rencontre et la connaissance de celles des autres, voire même d'adopter ce que nous ne préférons pas a priori, en « réfléchissant » nos propres préférences actuelles. C'est ce que l’on appelle le changement culturel (par exemple, en France, nous " préférons » aujourd'hui un mariage et une famille pour tous, plutôt qu'un mariage et une famille uniquement hétérosexuels).

Que ce soit dans la vie quotidienne ou bien dans la recherche en psychologie interculturelle, je rejoins alors tout à fait le mot de la fin rédigé par les auteurs:

Bien que la rencontre interculturelle soit une occasion de réflexion sur nos propres valeurs, voire de remise en question, elle n'implique en rien leur abandon. II n'y a donc pas de paradoxe à défendre ses propres valeurs tout en prônant le respect des différences culturelles. L'approche interculturelle consiste à tenir compte des différences culturelles de manière nuancée afin de mener à bien une action qui reste inspirée par des valeurs qui, le plus souvent, sont d'ailleurs partagées par les personnes issues d'autres cadres culturels. Adhérer à ses propres valeurs n'implique pas la non-reconnaissance des valeurs des autres. (p. 290)

\section{Références bibliographiques}

Bault, N., Chambon, V., Maïonchi-Pino, N., Pénicaud, F.-X., Putois, B. et Roy, J.-M. (dir.) (2011). Peut-on se passer de représentations en sciences cognitives? Bruxelles : De Boeck.

Berry, J., Poortinga, Y., Breugelmans, S., Chasiotis, A. et Sam, D. (2011). Cross-cultural psychology: Research and applications ( $3^{\mathrm{e}}$ éd.). New York : Cambridge University Press.

Bruner, J. (1991). ...Car la culture donne forme à l'esprit. De la révolution cognitive à la psychologie culturelle. Paris : EsHel. ( $1^{\mathrm{e}}$ édition en anglais, 1990)

Cole, M. (1996). Cultural psychology. A once and future discipline. Cambridge/London : Harvard University Press.

Dasen, P. et Mishra, R. (2013). Cultural differences in cognitive styles. Dans B. R. Kar (dir.), Cognition and brain development. Washington, DC : APA.

Alterstice - Revue Internationale de la Recherche Interculturelle, vol. $3, n^{\circ} 1$ 
De Greck, M., Shi, Z., Wang, G., Zuo, X., Yang, X., Wang, X., Northoff, G. et Han, S. (2012). Culture modulates brain activity during empathy with anger. Neurolmage, 59, 2871-2882.

Devereux, G. (1980). De l'angoisse à la méthode dans les sciences du comportement. Paris : Aubier. ( $1^{\mathrm{e}}$ édition anglaise, 1967)

Hagège, C. (2012). Contre la pensée unique. Paris : Odile Jacob.

Hayward, J. et Varela, F. (1995). Passerelles. Entretien avec le Dalaï-Lama sur les sciences de l'esprit. Paris : Albin Michel. ( $1^{\mathrm{e}}$ éd. en anglais, 1992)

Jahoda, G. (2002). The shifting sands of 'culture'. Dans P. Boski, F. Van de Vijver et M. Chodynicka (dir.), New directions in cross-cultural psychology (p. 91-106). Varsovie : Polish Psychological Association.

Laplantine, F. (1995). L'anthropologie. Paris : Payot \& Rivages. (1 ${ }^{\mathrm{e}}$ éd., 1987)

Le Pichon, A. et Sow, M. (dir.) (2011). Le renversement du ciel. Parcours d'anthropologie réciproque. Paris : CNRS Éditions.

Licata, L. et Heine, A. (2012). Introduction à la psychologie interculturelle. Bruxelles : De Boeck Université.

Nisbett, R. (2003). The geography of thought: How Asians and Westerners think differently, and why. Londres : Nicholas Brealey.

Popper, K. (1998). Des sources de la connaissance et de l'ignorance. Paris : Payot. (1 ${ }^{e}$ éd., 1963)

Schaller, M., Norenzayan, A., Heine, S., Yamaqishi, T. et Kameda, T. (dir.) (2010). Evolution, culture and the human mind. New York : Psychology Press.

Stigler, J., Shweder, R. et Herdt, G. (dir.) (1990). Cultural psychology. Essays on comparative human development. Cambridge : Cambridge University Press.

Troadec, B. (2003). Point de vue sur l'interculturel : les schèmes d'un psychologue du développement cognitif. Bulletin de Psychologie, 56(1), 463, 3-21.

Troadec, B. (2007). Constructivism, culture, and cognitive development. Which kinds of scheme for a cultural psychologist? Constructivist Foundations, 3(1), 38-51.

Troadec, B. (2011). Cognition et culture : le rôle de la transmission sociale et culturelle. Dans B. Troadec et T. Bellaj (dir.), Psychologies et cultures (p. 25-62). Paris : L'Harmattan.

Varela, F., Thompson, E. et Rosch, E. (1993). L'inscription corporelle de l'esprit. Sciences cognitives et expérience humaine. Paris : Seuil. 